\title{
Occupational activity and the risk of hip osteoarthritis
}

\author{
Cyrus Cooper, Lesley Campbell, Patricia Byng, Peter Croft, David Coggon
} \begin{abstract}
this review we shall focus on the relation between occupational physical activity and the risk of osteoarthritis of the hip.

MRC Environmental University of Southampton, Southampton, General
\end{abstract} Hospital, Southampton SO16 6YD, United Kingdom C Cooper L Campbell P Byng D Coggon

North Staffordshire Medical Institute, Industrial and Community Health Research Centre, Hartshill, Stoke-on-Trent ST4 7NY P Croft

Although the effect is weaker than with knee osteoarthritis, obesity is known to increase the risk of hip osteoarthritis. ${ }^{45}$ In one cross sectional population based study among men, those in the highest third of the distribution of adiposity had a 2.6-fold greater risk of
Osteoarthritis is probably the most common joint disorder in the world. In western populations, radiographic evidence of osteoarthritis occurs in the majority of people by the age of 65 years, and in about $80 \%$ of those aged 75 years and above. ${ }^{1}$ The disorder is second only to ischaemic heart disease as a cause of work related disability in men over 50 years of age, and accounts for more hospital admissions than rheumatoid arthritis each year.

Osteoarthritis is defined as focal loss of articular cartilage with variable subchondral bone reaction. There is incomplete concordance between these pathological features and radiographic or clinical characteristics of the disorder. However, the difficulties encountered in using a pathological definition for epidemiological studies of osteoarthritis have led to the widespread use of radiological and clinical markers. The radiographic features conventionally used to define disease severity include joint space narrowing, osteophytes, subchondral sclerosis, cyst formation, and abnormalities of bony contour. These radiographic features can be incorporated in rating scales at the commonly affected joint sites (for example the hand, knee, and hip) permitting standardisation of disease severity. ${ }^{2}$ The two clinical sequelae of osteoarthritis which are most relevant to epidemiological studies are joint pain and functional impairment. The development of osteoarthritis at any joint site depends upon a generalised predisposition to the condition, and abnormalities of biomechanical loading which act at specific joints. ${ }^{13}$ Individual risk factors which may be associated with a generalised susceptibility to the disorder including obesity, a family history, and hypermobility. Those which reflect local biomechanical insults include trauma, abnormalities of joint shape, and physical activity. In

developing hip osteoarthritis than those in the lowest third. ${ }^{4}$ The largest study to include women used 2490 subjects aged 55-74 years enrolled in the First National Health and Nutrition Examination Survey (NHANES-1). Among women, obesity was associated with bilateral but not unilateral hip osteoarthritis. ${ }^{5}$ The relation of hip osteoarthritis to obesity has also been observed in longitudinal studies, suggesting that the obesity antecedes the arthritis, ${ }^{6}$ rather than being a result of the inactivity induced by the hip disease. However, the extent to which obesity influences the progression of hip osteoarthritis remains less certain.

HIP INJURY

Several case series have documented a high frequency of hip osteoarthritis among people who have sustained lower limb injuries, particularly if these are severe enough to result in fracture or dislocation. ${ }^{7}$ Such trauma is associated with $5-10 \%$ of all cases of hip osteoarthritis and around $30 \%$ of patients who experience it develop osteoarthritis over a 20 year period. As with knee osteoarthritis, trauma appears to be associated more strongly with unilateral than with bilateral disease, and the relation is stronger among men than women. ${ }^{5}$

CONGENITAL AND DEVELOPMENTAL DISORDERS

Three distinct congenital and developmental disorders may occur in the hip joint: congenital dislocation, Perthes disease, and slipped femoral epiphysis. All three disorders result in an increased risk of later hip osteoarthritis, which tends to occur at relatively young ages (35-55 years) and to progress rapidly. However, the incidence of these three disorders is relatively low in the general population, and they are unlikely to account for more than a small proportion of hip osteoarthritis overall. It is possible that mild variants of each disorder, but particularly of acetabular dysplasia, also predispose to osteoarthritis. ${ }^{8}$ However, cross sectional studies exploring this hypothesis have been unable to confirm any association between radiographic measures of dysplasia and of hip osteoarthritis. Furthermore, populations in which shallow acetabula are more prevalent (such as the Japanese), appear to have lower rather than higher hip osteoarthritis rates than western populations. Prospective data relating acetabular dimensions early in life to the later risk of hip osteoarthritis will be required before this issue can be resolved.
CONSTITUTIONAL PREDISPOSITION

The fourth important risk factor for hip osteoarthritis is a constitutional predisposition 
to the disorder. The best clinical marker of such a predisposition is the presence of Heberden's nodes. Polyarticular involvement in osteoarthritis is unequivocal. However, the issue is whether the frequency of polyarticular osteoarthritis is greater than would be expected simply from the rising prevalence of osteoarthritis at each individual site with age. Recent population studies suggest that the clustering of joint involvement in osteoarthritis is appreciable, and that a polyarticular subset clearly exists, especially among postmenopausal women. ${ }^{9}$ Among the various joint sites of predilection (hand, knee, hip, and spine), the hip has the weakest association with the other three sites. Nevertheless, the presence of hand osteoarthritis appears to increase the risk of hip osteoarthritis around threefold. ${ }^{10}$

\section{Occupational activity and hip osteoarthritis}

The repetitive use and mechanical loading of certain joints appears to predispose to osteoarthritis. Thus for example cotton workers are prone to hand osteoarthritis and workers in occupations which entail repetitive or prolonged knee flexion are at increased risk of knee osteoarthritis. ${ }^{311}$ The contribution of mechanical stress to the risk of hip osteoarthritis is less certain. Detailed data on the relation between specific occupational activities, such as heavy lifting, and the risk of hip osteoarthritis are not currently available. However, evidence has emerged that one such occupation-farming - is associated with high rates of the disorder. The association was initially documented in a French ecological study suggesting higher arthroplasty rates in rural parts of the country. ${ }^{12}$ It has now been confirmed by Scandinavian and British data (table).

Axmacher and Lindberg reported that the prevalence of radiographic osteoarthritis among farmers who had undergone pelvic radiography for non-skeletal indications was almost 10 times higher than that expected among the male Malmo population of similar age. ${ }^{13}$ Thelin compared 105 cases listed for hip arthroplasty with population controls and found the risk for surgery to be 3.2 for men who had spent more than a decade in farming. ${ }^{14}$ Finally case-control and cohort

Agricultural labour and the risk of hip osteoarthrosis (OA)

\begin{tabular}{|c|c|c|c|}
\hline Study & Design & Sample size & $\begin{array}{l}{ }^{*} \text { Odds } \\
\text { ratio }\end{array}$ \\
\hline Louyot $(1966)^{12}$ & Survey & 2560 & 4.0 \\
\hline Typpo $(1985)^{9}$ & Case-control & 479 & 1.8 \\
\hline $\begin{array}{l}\text { Jacobsson } \\
(1987)^{20}\end{array}$ & Case-control & 262 & 3.0 \\
\hline $\begin{array}{c}\text { †Axmacher } \\
(1988)^{13}\end{array}$ & Survey & 309 & 10.6 \\
\hline Thelin $(1990)^{14}$ & Case-control & 327 & 3.2 \\
\hline Vingard $(1991)^{17}$ & Cohort & 35981 & 3.8 \\
\hline Croft $(1992)^{18}$ & Case-control & 539 & 2.0 \\
\hline tCroft $(1992)^{4}$ & Survey & 250 & 9.3 \\
\hline
\end{tabular}

* Odds ratio for hip OA among farmers/agricultural labourers, estimated from data.

† Prevalence ratios for hip OA among farmers compared with referent groups. studies based on the hospital discharge register of the Swedish National Board of Health and Welfare found that farmers, construction workers, food processing workers, and firefighters had excess risks of developing hip osteoarthritis resulting in hospital care..$^{15-17}$

However, farmers may obtain treatment more often than other occupational groups, not because they have a higher incidence of the disorder, but because they are more handicapped by it when it occurs. This issue may be addressed by population based radiographic studies comparing the prevalence of hip osteoarthritis between farmers and other occupational groups. In a British study ${ }^{4}$ comparing men from a defined population aged 60-75 years who had ever been farmers with a group of controls who had spent their entire careers in clerical work, the prevalence of moderate/severe radiographic hip osteoarthritis (defined as a minimal joint space of less than $1.5 \mathrm{~mm}$ ) was found to be $18 \%$ in the former group as compared with $2.4 \%$ in the controls. This gave a summary odds ratio for hip osteoarthritis among the farmers of 9.3 ( $95 \%$ confidence interval 1.8 to 33.8 ), a risk estimate comparable with the previously described Swedish study.

The precise reasons for the increased risk of hip osteoarthritis among farmers remain uncertain. It is currently unclear whether the excess risk might be found in other heavy manual workers, for example construction workers and labourers. In a second British case-control study, ${ }^{18}$ the risk of hip osteoarthritis was found to be related to occupations which entailed regular heavy lifting (for example, the daily moving of weights greater than 25 $\mathrm{kg}$ by hand), prolonged standing, and walking over rough ground. However, the magnitude of the risk associated with these occupational exposures was substantially less than that found in the studies comparing farmers with other occupational groups, and further research into the nature of the farming/hip osteoarthritis relationship is required.

\section{Conclusion}

There is now clear epidemiological evidence that occupational activity is a contributor to the risk of osteoarthritis at the hip and knee. The pattern of activity which predisposes to osteoarthritis at these two joint sites appears to be different. For the knee, evidence suggests that repetitive knee use, perhaps coupled with heavy lifting, is the principal biomechanical risk factor. The risk of knee osteoarthritis is found to be increased in several occupations characterised by these exposures. Studies are consistent in documenting an increase in risk of hip osteoarthritis among agricultural workers, but the precise mechanism for this association remains the subject of study. Although the weight of current evidence may not be sufficient to tip the balance of probabilities in favour of permitting recompense to workers in these occupations who develop osteoarthritis, this matter has begun to receive scrutiny from the appropriate occupational health authorities in several western nations. 
These studies were supported by project grants from the Arthritis and Rheumatism Council of Great Britain, the Wellcome Foundation, and the Medical Research Council. The manuscript was typed by Miss Katy Cuninghame.

1 Cooper C. The epidemiology of osteoarthritis. In: Klippel J, Dieppe P, eds. Rheumatology. New York: CV Mosby, 1994:7.3.1-4.

2 Spector TD, Cooper C. Radiographic assessment of osteoarthritis: whither Kellgren and Lawrence? Osteoarthritis Cartilage 1993;1:203-6.

3 Felson DT. Epidemiology of hip and knee osteoarthritis. Epidemiol Rev 1988;10:1-28.

4 Croft P, Coggon D, Cruddas M, Cooper C. Osteoarthritis of the hip: an occupational disease in farmers. $B M F$ 1992;304:1269-72.

5 Tepper, S. Hochberg MC. Factors associated with hip osteoarthritis: data from the First National Health and Nutrition Examination Survey (NHANES-1). Am $\mathcal{f}$ Epidemiol 1993;137:1081-8.

6 Van Saase JLCM. Osteoarthrosis in the general population: a follow-up study of osteoarthrosis of the hip. Rotterdam: Erasmus University, 1989 (dissertation)

7 Lequesne M, Azorin M, Lamotte J. Post-traumatic osteoarthritis of the hip. Rev Rhum 1993;60:698-704.

8 Croft P, Cooper C, Wickham C, Coggon D. Osteoarthritis of the hip and acetabular dysplasia. Ann Rheum Dis 1991; 50:308-10.

9 Egger P, Cooper C, Hart D, Doyle D, Coggon D, Spector T. Patterns of joint involvement in osteoarthritis of the hand: the Chingford Study. $\mathcal{F}$ Rheumatol 1995;22:1509- 13.
10 Croft P. Cooper C, Wickham C, Coggon D. Is the hip involved in generalised osteoarthritis? $\mathrm{Br} \mathcal{F}$ Rheumatol 1992;31:325-8.

11 Cooper C, McAlindon T, Coggon D, Egger P, Dieppe P. Occupational activity and osteoarthritis of the knee. Ann Rheum Dis 1994;53:90-3.

12 Louyot P, Savin R. La coxarthrose chez l'agriculteur. Rev Rhum 1966;33:625-32.

13 Axmacher $B$, Lindberg $H$. Coxarthrosis in farmers as appearing on colon radiograms and urograms. In: Hogstedt C, Reuterwall C, eds. Progress in occupational epidemiology. Amsterdam: Excerpta Medica, 1988:203-6.

14 Thelin A. Hip joint arthrosis: an occupational disorder among farmers. Am f Ind Med 1990;18:339-43.

15 Vingard E. Work, sports, overweight and osteoarthrosis of the hip. Stockholm: Karolinska Institute, 1991 (dissertation).

16 Vingard E, Hogstedt C, Alfredsson L, Fellenius E, Goldie I, Koster M. Coxarthrosis and physical work load. Scand $\mathcal{f}$ Work Environ Health 1991;17:104-9.

17 Vingard E, Alfredsson L, Goldie I, Hogstedt C. Occupation and osteoarthrosis of the hip and knee: a register-based cohort study. Int f Epidemiol 1991;20:1025-31.

18 Croft P, Cooper C, Wickham C, Coggon D. Osteoarthritis of the hip and occupational activity. Scand $\mathcal{F}$ Work Environ Health 1992;18:59-63.

19 Typpo T. Osteoarthritis of the hip. Acta Chir Gynaecol 1985; 74(suppl 201):1-38.

20 Jacobsson B, Dalen N, Tjornstrand B. Coxarthrosis and labour. Int Orthop 1987;11:311-3.

\title{
Physical activity at leisure and risk of osteoarthritis
}

\author{
Nancy E Lane
}

Exercise, particularly weight bearing sports like running and team sports, remains popular throughout the industrialised world. Evidence supports participating in regular exercise, including recreational activities or competitive sports, as it improves general health and may increase longevity. ${ }^{12}$ Therefore increasing numbers of individuals have chosen to pursue regular exercise programmes. Individuals with normal joints ask whether their exercise programmes increase the risk of developing osteoarthritis, and individuals with osteoarthritis of weight bearing joints ask what types of physical activity might accelerate the progression of their joint disease. Since osteoarthritis is the major cause of activity limitation in the elderly, these questions are relevant public health issues.

In this paper I shall review the studies that address exercise as a risk factor for osteoarthritis in normal and abnormal joints and suggest future studies which may further our understanding of this issue.

\section{Normal joints}

LOW IMPACT

Several studies have examined the relation of jogging to osteoarthritis. ${ }^{3-11}$ While some of the retrospective cross sectional studies show no impact of exercise on osteoarthritis in normal joints ${ }^{3-59}$ others do ${ }^{1011}$.

In 1984, Lane et al initiated a longitudinal study to examine the association of jogging with the development of osteoarthritis and musculoskeletal disability in a group of runners over the age of $\mathbf{5 0}$ years and controls.
Runners were compared with 41 control subjects matched for age, sex, years of education, and occupation. ${ }^{3}$ The results of this initial cross sectional study showed that women runners, at a mean age of 59 years and an average of nine years of running over 200 minutes a week, had more radiographic evidence of knee subchondral sclerosis and osteophytes than controls. However, no increase in self reported knee joint pain or clinical osteoarthritis was found. Male runners were not different in either radiographic or clinical evidence of osteoarthritis from controls. ${ }^{3}$ At the five year follow up, a subgroup of the original cohort was examined. These subjects (mean age 65 years) continued to run 180 minutes per week. ${ }^{7}$ Both runners and controls had significant radiographic progression of the individual radiographic features of osteoarthritis. Five controls and four runners had knee osteoarthritis by the American College of Rheumatology criteria. ${ }^{7}$ Predictors of the radiographic knee osteoarthritis score included age, pace per mile, and weight. ${ }^{7}$ At the nine year follow up, 32 of the original 41 runners and 23 controls were examined (mean age 67 years). In 1993 the radiographic scores for knee osteoarthritis were similar for those still running, those who had stopped running, and those who had never run. ${ }^{12}$

Similar observations were reported by Panush et al. When 17 male runners (mean age 56 years) were compared with age and weight matched controls, ${ }^{4}$ no differences in radiographic or clinical hip, knee, or ankle osteoarthritis were found. An eight year follow up of a subgroup of the original cohort found that both runners and controls had some

\author{
Rheumatology \\ University of \\ Francisco, San \\ Francisco, California \\ 94143, USA \\ N E Lane
}

